\title{
NUTRITIONAL REQUIREMENT AMONG PSEUDOMONAS AERUGINOSA ISOLATES RECOVERED FROM RESPIRATORY CLINICAL SPECIMENS AT A TERTIARY HOSPITAL FROM SOUTH OF BRAZIL
}

\author{
Leandro Reus Rodrigues Perez ${ }^{*}$; Ana Lúcia Peixoto de Freitas ${ }^{2}$; Afonso Luís Barth ${ }^{2,3}$
}

\begin{abstract}
${ }^{1}$ Programa de Pós-Graduação em Ciências Farmacêuticas, Faculdade de Farmácia, Universidade Federal do Rio Grande do Sul, Porto Alegre, RS, Brasil; ${ }^{2}$ Departamento de Análises Clínica, Faculdade de Farmácia, Universidade Federal do Rio Grande do Sul, Porto Alegre, RS, Brasil; ${ }^{3}$ Serviço de Patologia Clínica, Hospital de Clínicas de Porto Alegre, Porto Alegre, RS, Brasil.
\end{abstract}

Submitted: August 02, 2010; Returned to authors for corrections: October 15, 2010; Approved: January 31, 2011.

\begin{abstract}
We screened 349 isolates of $P$. aeruginosa from cystic fibrosis (CF+) and non-cystic fibrosis (CF-) patients for the auxotrophy. Fourteen $(4.0 \%)$ were auxotrophic and among them only one was recovered from CFpatient showing that this characteristic is strongly associated with cystic fibrosis. In total, a requirement for 5 different compounds (or combination) was verified and, of these, methionine was the most common single amino acid required. Only one auxotrophic isolate was no able to produce biofilm in vitro.
\end{abstract}

Key words: Pseudomonas aeruginosa, auxotrophy, cystic fibrosis, biofilm

Pseudomonas aeruginosa is the most common organism isolated from the respiratory tract of patients with cystic fibrosis (CF). Once established in the airways of patients with $\mathrm{CF}, P$. aeruginosa undergoes some modifications to adapt and persist in this environment. Some isolates of $P$. aeruginosa from patients with $\mathrm{CF}$ also have specific nutritional requirements and they are called auxotrophic because of their inability to grow in a basic medium containing mineral salts and glucose as the sole carbon source (3).

Generally, $P$. aeruginosa from clinical and environmental sources are prototrophic, but approximately half of CF patients colonized with $P$. aeruginosa are harboring isolates that require the provision of specific factors for growth $(3,4)$. The most common nutritional requirement of these auxotrophic isolates is methionine, but other amino acids such as leucine, arginine, or ornithine may also be required by a minority of isolates. Sometimes, auxotrophic isolates are also found in patients with bronchiectasis, but they have not been isolated from patients with other conditions (1). Difficulty for eradication and antimicrobial resistance are characteristics presented by auxotrophic isolates that have been related to its easy adaptation inside lung with $\mathrm{CF}$ and the ability to produce biofilm (1).

The aim of the study presented here was to evaluate the nutritional requirement for $P$. aeruginosa isolates recovered from sputa and endotracheal aspirate of $\mathrm{CF}+$ and $\mathrm{CF}$ - patients. Furthermore, we evaluated the ability of biofilm production for the auxotrophic isolates.

*Corresponding Author. Mailing address: Faculdade de Farmácia, Universidade Federal do Rio Grande do Sul (UFRGS) Avenida Ipiranga, 2752, 90610-000, Porto Alegre, RS, Brazil.; E-mail: leandro.reus@gmail.com 
A total of $349 P$. aeruginosa isolates were included in the study, $165(47.3 \%)$ and $184(52.7 \%)$ isolates from $\mathrm{CF}+$ and CF- patients, respectively. Non-CF hospitalized patients had no record of bronchiectasis documented. All isolates were identified by using a NC32 panel and WalkAway ${ }^{\circledR}$ 96SI automated system (Siemens, Newark, DE, USA) for reading. Also, Gram stain, oxidase test and colony morphology were concomitantly inspected. All isolates were recovered from respiratory clinical specimens (endotracheal aspirate and sputum). More than one isolate from the same culture could be included in the study once that occurrence of auxotrophic and prototrophic isolates has already been concomitantly described.

A 0.5 McFarland suspension of each isolate was diluted $1: 10$ in saline. An aliquot of $0.3 \mu \mathrm{L}$ of the suspension was applied onto Mueller-Hinton agar (complete medium) and onto minimal agar medium (MAM) just containing mineral salts and glucose (minimal medium) using a Steers multipoint inoculator. The plates were incubated at $37^{\circ} \mathrm{C}$ and were screened after $48 \mathrm{~h}$. Prototrophic isolates were able to grow in both media while auxotrophic isolates were unable to grow on MAM. Two methods were used to verify the amino acid nutritional requirement. Firstly, MAM plates individually containing $20 \mu \mathrm{g} / \mathrm{mL}$ one of the 23 amino acids (L-forms of alanine, arginine, asparagine, aspartic acid, cysteine, cystine, glutamic acid, glutamine, glycine, histidine, hydroxy-L-proline, isoleucine, leucine, lysine, methionine, ornithine, phenylalanine, proline, serine, threonine, tryptophan, tyrosine, and valine; Sigma Chemical Co., St. Louis, Mo.) were inoculated as described above. Growth in the presence of a specific amino acid indicates that the bacteria need this component, while the failure to grow in this condition suggests that one or more amino acids in combination or a distinct growth factor is needed. Secondly, all amino acids less one were added into MAM plates. The specific requirement of one or more amino acid is evident when the isolate is unable to grow on medium without this amino acid.

The ability to produce biofilm for the auxotrophic isolates was determined by microtiter plate assay according to the protocol previously described (5).

Of the 349 isolates evaluated, 14 (4\%) were auxotrophic. Among these auxotrophic isolates, only one isolate were recovered from a CF- patient. Table shows that among the 14 auxotrophic strains identified, the most common single amino acid required was methionine ( 8 isolates), followed by arginine and asparagine (2 isolates each). One isolate grew only on a combination of alanine and proline, and other required a combination of methionine, cysteine and threonine (Table 1). Because the presence of some amino acids are required in order to allow the growth of auxotrophic isolate, it is possible that these amino acids are present in the airways of CF patients. $P$. aeruginosa wild-type are nutritionally versatile. Thus, we speculate that this would be the original mode of the respiratory tract colonization and that wild-type isolate would be substituted by its auxotrophic variants during the infection process (3). The selection process of auxotrophic isolates in the lung is not clear; however it is known to be a bacterial strategy to prevent the waste of energy through downregulation of a metabolic way, in view to reduce the production of some substances freely present in the environment. Synthesis of amino acid is energetically expensive and bacteria with loss of ability to produce some amino acid would have metabolic and energetic advantages (7).

Some studies have shown that auxotrophic $P$. aeruginosa isolates are highly associated with acute exacerbations of respiratory infection in $\mathrm{CF}$ patients $(2,3,4)$, and that these isolates are often more resistant to antimicrobial agents than prototrophic wild-type isolates (2). This antimicrobial resistance can be explained, in part, due to the biofilm production, once that there is a microenvironment in the $\mathrm{CF}$ lung that permit the ideal condition for the biofilm formation. Indeed, in our study, thirteen of the 14 auxotrophic strains presented weak- (10 isolates) and moderate- (3 isolates) biofilm production in vitro (Table 1). Resistance rates for the auxotrophic isolates were of $57.1 \%$ to ciprofloxacin; $35.7 \%$ to 
gentamicin; $28.6 \%$ to amikacin, aztreonam, cefepime and ceftazidime; $21.4 \%$ to imipenem; and $14.3 \%$ to meropenem and piperacillin/tazobactam. One isolate, auxotrophic to arginine, showed resistance to all antibiotics tested except polimixin (data not shown).

In this study, the most required amino acids were methionine and arginine, which were previously reported as the most common ones for the auxotrophic isolates $(1,4)$. High level serum of the key-precursor of methionine, called sulphadenosylmethionone, is verified in CF patients (6). Thus, methionine synthetase inhibitors and antimicrobial therapy together could be useful for the treatment of these patients (6).
To our knowledge, this is the first description of auxotrophy phenomenon among Brazilian isolates of $P$. aeruginosa from CF patients. On the other hand, a potential limitation of this study lies in the fact that the genetic background of the isolates was not evaluated and, thus, the origin of auxotrophic from prototrophic mutants can not be clearly defined.

Finally, we conclude that methionine was the main nutritional requirement among auxotrophic isolates and that this phenomenon was more frequently observed in $P$. aeruginosa recovered from sputa of $\mathrm{CF}$ patients indicating that this is a characteristic of this bacteria in this group of patients.

Table 1. Nutritional requirement and biofilm production among 14 auxotrophic Pseudomonas aeruginosa isolates.

\begin{tabular}{llll}
\hline Isolate number & Cystic fibrosis & Nutritional requirement & Biofilm production \\
\hline 86 & yes & methionine & no-producing \\
101 & yes & methionine & weak \\
144 & no & methionine & weak \\
146 & yes & methionine & weak \\
165 & yes & arginine & weak \\
178 & yes & arginine & weak \\
197 & yes & alanine and proline & weak \\
214 & yes & asparagine & moderate \\
233 & yes & asparagine & moderate \\
236 & yes & methionine & weak \\
338 & yes & methionine & moderate \\
340 & yes & methionine & weak \\
342 & yes & methionine & weak \\
345 & yes & methionine, cysteine and threonine & weak \\
\hline${ }^{a}$ Classification of biofilm production determined by microtiter plate assay according to Stepanovic et al $(6)$.
\end{tabular}

${ }^{a}$ Classification of biofilm production determined by a microtiter plate assay according to Stepanovic et al (6).

\section{ACKNOWLEDGEMENTS}

The authors are grateful to Marcelo Dutra Arbo and Ana Lúcia Souza Antunes for the technical support.

\section{REFERENCES}

1. Agarwal, G.; Kapil, A.; Kabra, S.K.; Das, B.K.; Dwivedi, S.N. (2005). Characterization of Pseudomonas aeruginosa isolated from chronically infected children with cystic fibrosis in India. BMC Microbiol. 43 (5),
43-54.

2. Anzaudo, M.M.; Busquets, N.P.; Ronchi, S.; Mayoral, C. (2005). Microorganismos patógenos aislados en muestras respiratorias de niños con fibrosis quística. Rev. Argent. Microbiol. 37 (3), 129-134.

3. Barth, A.L.; Pitt, T.L. (1995). Auxotrophy of Burkholderia (Pseudomonas) cepacia from cystic fibrosis pacients. J. Clin. Microbiol. 33 (8), 2192-2194.

4. Barth, A.L.; Woodford, N.; Pitt, T.L. (1998). Complementation of methionine auxotrophs of Pseudomonas aeruginosa from cystic fibrosis. Curr. Microbiol. 36 (4), 190-195.

5. Stepanovic, S.; Vucovic, D.; Hola, V.; Bonaventura, G.; Djukic, S.; 
Irkovic, I.C.; Ruzicka, F. (2007). Quantification of biofilm in microtiter plates: overview of testing conditions and practical recommendations for assessment of biofilm production by staphylococci. APMIS 115 (8), 891899.

6. Taylor, R.F.H.; Hodson, M.E.; Pitt, T.L. (1993). Adult cystic fibrosis: association of acute pulmonary exacerbations and increasing severity of lung disease with auxotrophic mutants of Pseudomonas aeruginosa. Thorax 48 (10), 1002-1005.

7. Thomas, S.R.; Ray, A.; Hodson, M.E.; Pitt, T.L. (2000). Increased sputum amino acid concentrations and auxotrophy of Pseudomonas aeruginosa in severe cystic fibrosis lung disease. Thorax 55 (9), 795797. 